\title{
Pneumatic Networks for Soft Robotics that Actuate Rapidly
}

\section{Citation}

Mosadegh, Bobak, Panagiotis Polygerinos, Christoph Keplinger, Sophia Wennstedt, Robert F. Shepherd, Unmukt Gupta, Jongmin Shim, Katia Bertoldi, Conor J. Walsh, and George M. Whitesides. 2014. "Pneumatic Networks for Soft Robotics That Actuate Rapidly." Advanced Functional Materials 24 (15) (January 10): 2163-2170. doi:10.1002/adfm.201303288.

\section{Published Version}

doi:10.1002/adfm.201303288

\section{Permanent link}

http://nrs.harvard.edu/urn-3:HUL.InstRepos:25922120

\section{Terms of Use}

This article was downloaded from Harvard University's DASH repository, and is made available under the terms and conditions applicable to Open Access Policy Articles, as set forth at http:// nrs.harvard.edu/urn-3:HUL.InstRepos:dash.current.terms-of-use\#OAP

\section{Share Your Story}

The Harvard community has made this article openly available.

Please share how this access benefits you. Submit a story.

\section{Accessibility}




\section{Pneumatic Networks for Soft Robotics that Actuate Rapidly}

Bobak Mosadegh ${ }^{1,2}$, Panagiotis Polygerinos ${ }^{3}$, Christoph Keplinger ${ }^{1}$, Sophia Wennstedt ${ }^{1}$, Robert

F. Shepherd ${ }^{1}$, Unmukt Gupta ${ }^{1}$, Jongmin Shim ${ }^{3}$, Katia Bertoldi ${ }^{3}$, Conor J. Walsh ${ }^{2,3}$, and George M. Whitesides ${ }^{1,2^{*}}$

${ }^{1}$ Department of Chemistry and Chemical Biology, Harvard University, 12 Oxford Street, Cambridge, MA 02138, USA.

${ }^{2}$ Wyss Institute for Biologically Inspired Engineering, Harvard University, 60 Oxford Street, Cambridge, MA 02138, USA.

${ }^{3}$ School of Engineering and Applied Sciences, Harvard University, Cambridge, MA 02138, USA.

(*) Correspondence should be addressed to: gwhitesides@gmwgroup.harvard.edu

Target Journal: Advanced Functional Materials 


\section{Pneumatic Networks for Soft Robotics that Actuate Rapidly}

\section{Abstract}

Soft robots actuated by pressurization and inflation of a pneumatic network (a "pneunet") of small channels in elastomeric materials are appealing for their ability to produce sophisticated motions with simple controls. Although current designs of pneu-nets achieve motion with large amplitudes, they do so relatively slowly (that is, over seconds). This paper describes a new design for pneu-nets that reduces the amount of gas that must be transported for inflation of the pneu-net, and thus increases its speed of actuation. A simple actuator can bend from a linear shape to a quasi-circular shape in 50 milliseconds when pressurized at $\Delta \mathrm{P}=$ $345 \mathrm{kPa}$. At high rates of pressurization and inflation, the path along which the actuator bends depends on this rate. When inflated fully, the channels and chambers of this new pneu-net design experience only one-tenth the change in volume of that required for a motion of equal amplitude using the previous design. This small change in volume requires comparably low levels of strain in the material at maximum amplitudes of actuation, and commensurately low rates of fatigue and failure. This actuator can operate over a million cycles without significant degradation of performance. This design for soft robotic actuators combines high rates of actuation with high reliability of the actuator, and opens new areas of application for them. 


\section{Introduction}

Elastomeric actuators powered pneumatically are of particular interest for soft robotics because they can be lightweight, inexpensive, easily fabricated, and able to provide non-linear motion with simple inputs ${ }^{[1-7]}$. Pneu-nets (networks of small channels embedded in elastomeric structures that can be inflated with low pressures-approximately $50 \mathrm{kPa}$-of air) usually require significant changes in volume $(\Delta V / V>20)$ to achieve their full range of bending. This requirement for large values of $\Delta \mathrm{V} / \mathrm{V}$ limits the performance of soft actuators that use pneu-nets in three ways: i) It requires the transfer of large volumes of gas for actuation. As a result, the rate of actuation is slow). ii) It generates a change of volume of the actuator that is significant. As a result, it requires that the system be surrounded by a large volume of empty space for it to operate. iii) It imposes high strains on the material of which the pneu-net is fabricated. As a result, the operating lifespan of the pneu-net is shortened. To circumvent these limitations, we have developed a new design for a pneu-net that is better suited for highspeed, large-amplitude motion than the design described previously ${ }^{[1-7]}$. The new actuator bends completely-from a linear rod $(10 \mathrm{~cm})$ into an approximate circle-at speeds greater than $1 \mathrm{~m} / \mathrm{s}$ for the tip of the actuator, and in intervals as short as $50 \mathrm{~ms}$.

This silicone-based actuator also has two other features: i) it is sufficiently durable that it can sustain intermediate-frequency $(4 \mathrm{~Hz})$, large-amplitude motion for greater than a million repeated cycles, without failure. ii) It shows a new mode of actuation. At low rates of inflation, it bends into approximately a circle with all chambers inflating uniformly; at high rates of inflation, it curls upon itself with the chambers at its tip inflating first. To mimic the speed, if 
not the force, of a moving human finger, we fabricated a set of pneu-nets that played a tune on an electric piano.

\section{Background}

Soft robots are machines fabricated from compliant materials (polymers ${ }^{[1-8]}$, elastomers ${ }^{[1]}$, hydrogels ${ }^{[9,10]}$, granules $\left.{ }^{[11]}\right)$; they can operate with several different modes of actuation (i.e., pneumatic ${ }^{[1,5,11-13]}$, electrical ${ }^{[7,14-18]}$, chemical ${ }^{[12,19,20]}$ ), and their motion can be either fast $(>1 \mathrm{~Hz})^{[9,12,14]}$ or slow $(<0.1 \mathrm{~Hz})^{[1,21]}$.

Exploration of rapid motion in soft robotics is just beginning, and examples are at a level of initial demonstrations ${ }^{[1,9,12,14]}$. For example, the "GoQBot" developed by Trimmer et al., is a worm-like robot that performs a single rapid actuation ( $<100 \mathrm{~ms})$ to achieve a ballistic rolling motion by using the deformation of coils of shape-memory alloy (SMA $)^{[14]}$. Although the body of the robot is made of soft materials, the rigidity of the coils of SMA restricts the movement of the robot to a single trajectory. In contrast, pneu-nets use a non-rigid actuator (i.e., inflatable channels fabricated in an elastomeric material) that allows its trajectory of motion (i.e., the shape it bends into) to change when encountering different external forces ${ }^{[1,4,5]}$. It is the compliance of the materials used for pneu-nets, however, that contributes to their three major weaknesses, i) slow actuation speed, ii) large change in volume, and iii) short lifespan.

High-speed actuation of a tripedal robot has been accomplished using the explosion of a mixture of methane and oxygen in a pneu-net, to generate a rapid (msec) pulse of pressure, and power a jumping motion $(>30 \mathrm{~cm} \text { at } 3.6 \mathrm{~m} / \mathrm{s})^{[12]}$. Although this explosion produced rapid 
motion, it is not presently adaptable to give precise control of the movement of the robot. Combustion is also not a suitable source of power for applications such as medicine. Soft robotics, therefore, need pneu-nets that actuate rapidly $(>1 \mathrm{~Hz})$, over their full range of motion, with conventional, safe methods.

\section{Results}

\subsection{Experimental Design}

The angular speed of bending of a structure actuated by a pneu-net depends upon: i) the rate of inflation, ii) the geometry of the internal channels and exterior walls, and iii) the properties of the structure (i.e., materials used for fabrication). We use pneumatic actuation, since pressurized air has four advantages: i) it provides rapid inflation of the pneumatic structure (because air has low viscosity and can be moved rapidly); ii) it is easily controlled and measured (using regulators and sensors); iii) it is almost universally available (either from compressed gas tanks or compressors); iv) it is light in weight; iv) it can be discarded after use by venting to the atmosphere. Because it is easy to fabricate prototype pneu-nets, we tested the new geometry empirically rather than by modeling it. We did, however, clarify and visualize strains in these systems during actuation, using simulations based on finite-element models (FEMs). We fabricated all of the actuators using silicone-based elastomers, since they are commercially available, easy to work with, and able to tolerate large strains (> $700 \%)$.

\subsection{Design of Rapidly Actuating Pneu-Nets}


We have previously described actuators based on pneu-nets ${ }^{[1,5,6]}$. These systems have consisted, in general, of an extensible top layer and an inextensible but flexible bottom layer (Fig. 1A); in this paper, we will refer to this type of structure as a "slow pneu-net" (sPN); but the abbreviation can also refer to "simple pneu-net" since the design and fabrication of these systems is slightly simpler than the ones we describe here. The extensible layer of a sPN contains chambers connected by a single channel that, when pressurized, preferentially expand the top, and stretch the inside walls, of the chambers. This change in shape results in the bending of the entire actuator (due to the difference in the compliance of the extensible and the inextensible layers). The maximum change in volume of the channels inside the pneu-nets due to deformation of the elastomeric material defines the total amount of compressed gas that must be transported into it to achieve full bending; larger changes in volume require longer durations of pressurization to reach complete actuation.

To reduce changes in the volume required for complete actuation, and thus to increase the rate of actuation, we designed a new actuator, which we refer to as a "fast pneu-net" (fPN) (Fig. 1B); the supplemental information describes its fabrication. The fPN, like the sPN, consists of an extensible top layer and inextensible, but flexible, bottom layer. In contrast to the sPN, the extensible layer of the fPN contains gaps between the inside walls of each chamber. We designed the dimensions of the chambers so that the two inside walls are thinner, and have greater surface area, than the other exterior walls. An increase in the internal pressure, therefore, preferentially expands the inside walls, and minimizes the strain that occurs on the other exterior walls. In addition, the close proximity of two neighboring chambers causes the 
Figure 1. Design of Slow and Fast Pneu-nets. A-B) Schematic of the slow pneu-net (sPN) (A) and fast pneu-net (fPN) (B) actuators each consists of an extensible top layer, and an inextensible bottom layer reinforced with embedded paper. The top layer of the sPN actuator contains topographical features only along the inside surface of the actuator. The fPN actuator contains topographical features along the inside and outside surfaces of the actuator. Solid arrows identify regions of the actuators, and dashed arrows signify expanding regions when the actuator is pressurized. 


\section{A Slow Pneu-net (sPN)}

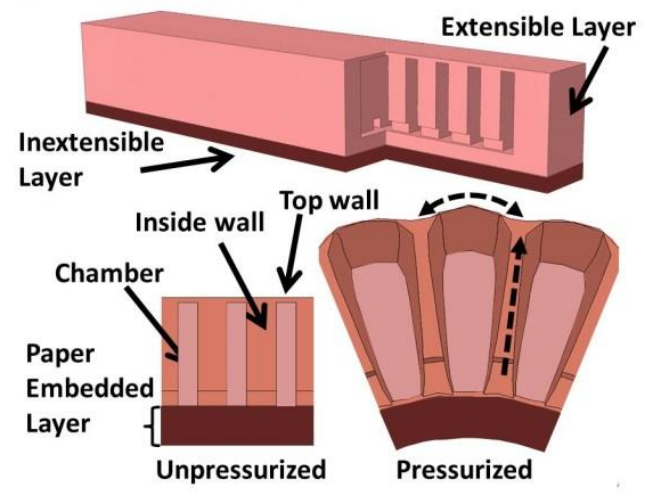

B Fast Pneu-net (fPN)

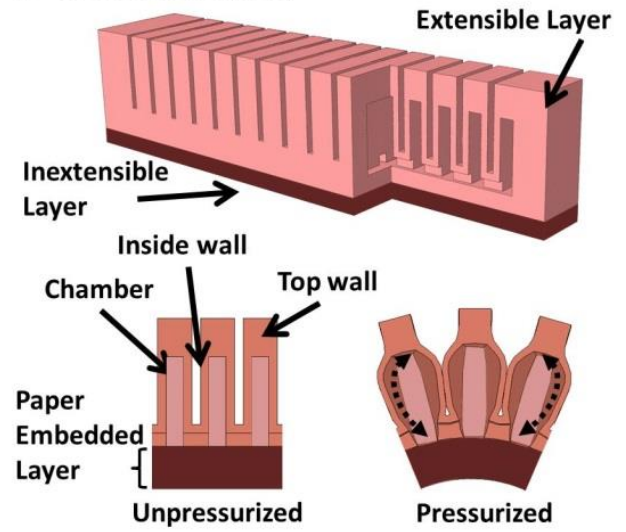


expanding inside walls to push against each other, and results in a preferential elongation of the extensible layer with only small changes $(<1 \%)$ in the height of this layer.

\section{3 fPNs of Different Dimensions}

To investigate the impact that different dimensions have on the bending of the fPN, we characterized the pressure required to bend it for different numbers of chambers, heights of chambers, and wall thicknesses of the chambers (Fig. S2). Empirically we determined that more chambers for a given length and thinner inside walls enabled greater bending at lower pressures (Fig. S2). The extent to which these trends can be exploited, however, is limited both by the properties of the materials, and by the fabrication of the pneu-nets. The resolution of the 3D printer ( $300 \mu \mathrm{m}$ for the 3D printers used in this work), used to make the molds determines the number of chambers and thickness of the walls that can be fabricated using this mold. The effect of increasing the height of a chamber, for a given width, appears to plateau, and therefore even taller chambers would only increase the actuator size, weight, and the amount of elastomer used without increasing the performance of the actuator (Fig. S2A). Although these trends suggest a guideline for designing fPNs, further work-both experimental and computational-will improve the performance of this class of actuators by exploring a larger volume of parameter space than that tested in this work ${ }^{[22]}$.

\subsection{Mass Transport Analysis of sPN and fPN}

To quantify the amount of fluid (normally air, but occasionally water during characterization) transported during actuation, we measured pressure-volume (PV) hysteresis curves for a test beam containing either a SPN or a fPN, with both submerged in water to 
counteract the force of gravity. We correlated these measurements with the extent of bending using continuous video recordings (Fig. 2AB). A syringe pump (Harvard Apparatus PHD 2000) transferred water (an incompressible fluid) into the actuator at a rate of inflation and deflation $(0.07614 \mathrm{~mL} / \mathrm{s})$ that was sufficiently slow to achieve quasi-static conditions. The incompressibility of the fluid allowed us to equate the volume decrease/increase of fluid in the syringe to that of the increase/decrease in the volume of the channels in the pneu-net. A pressure transducer, connected directly between the actuator and the syringe pump, monitored the pressure inside the system continuously (Fig. S3). Each PV hysteresis curve displays the pressure and volume levels necessary to achieve full bending (that is, sufficient bending that the two ends of the actuator touch) and deflation back to the initial state.

Six parameters describing the performance of the actuators can be extracted from the PV hysteresis plots generated using this procedure: i) the pressure of fluid required for full actuation; ii) the volume of fluid required for full actuation; iii) the input energy required for full bending of the actuator; iv) the energy that can be recovered during depressurization; v) the energy dissipated during one cycle of actuation; and vi) the shape (linearity or type of nonlinearity) of the PV curve. The maximum values for the $x$ - and $y$-axis signify the pressure and volume required for full bending of the actuator. The area below the inflation curve signifies the input energy of the actuator. The area below the deflation curve signifies the energy recovered from the actuator. The dissipated energy equals the difference between the input and recovered energies. 
Figure 2. Comparison of fPN and sPN actuators. A, B) Pressure-volume hysteresis curves for actuators undergoing quasistatic hydraulic inflation/deflation. The areas under the inflation and deflation curves signify the amount of energy added to and recovered from the actuator. Lost energy is the difference between the input and recovered energy. Inset images show actuators at the maximum inflation pressure. C-F) Simulated images, generated by a finiteelement model, of the bending of the $\operatorname{SPN}(C, E)$ and $f P N(D, F)$ actuators and the levels of the maximum principal strain (i.e., 1D dimensionless units) experienced by the actuator when fully pressurized. 
A sPN Actuator

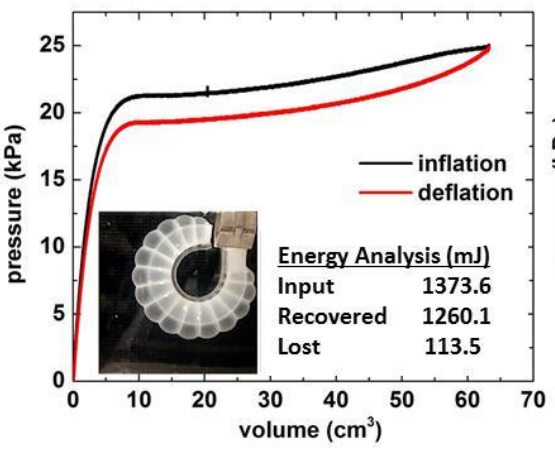

C sPN Actuator

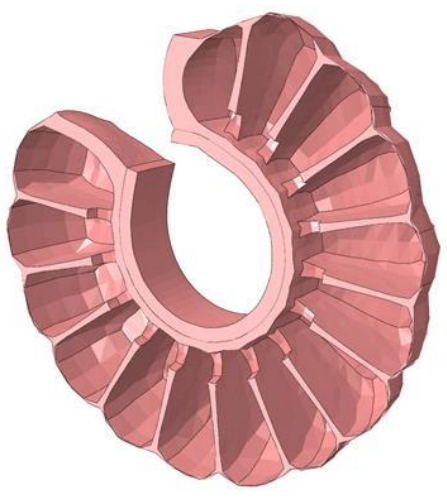

E

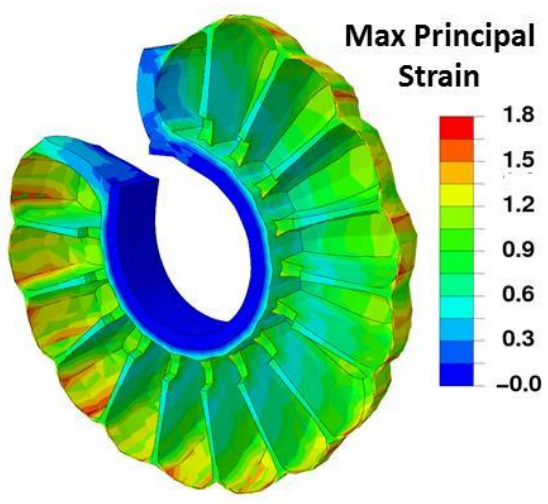

B fPN Actuator

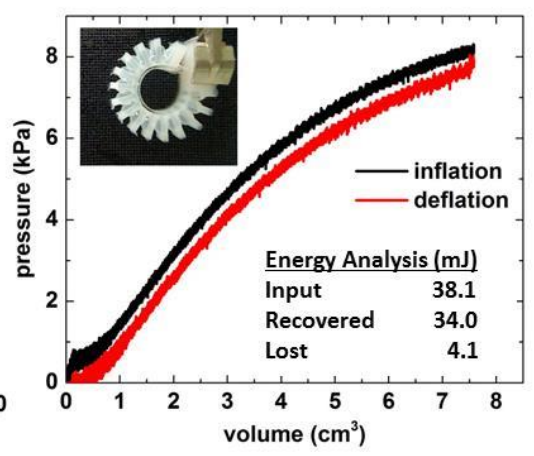

D fPN Actuator

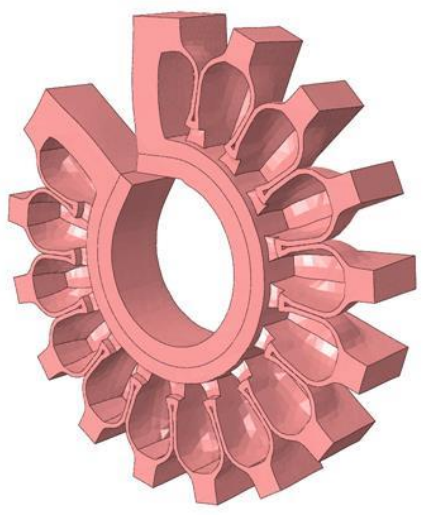

F fPN Strain

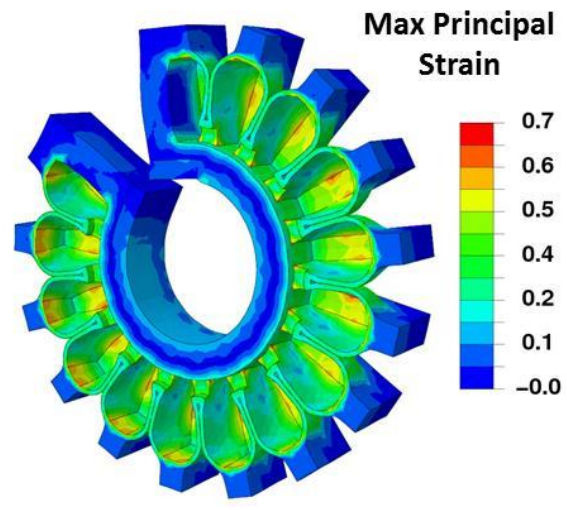


To compare the sPN and fPN design, we tested actuators made from Ecoflex 30 (for the extensible layer) and Sylgard 184 PDMS (for the inextensible layer). When using Ecoflex 30/PDMS as the elastomer, the results show the SPN requires $\sim 3 \mathrm{X}$ higher pressure, $\sim 8 \mathrm{X}$ higher volume, $35 \mathrm{X}$ more energy to bend fully than the fPN (Fig. 2AB). In addition, during one actuation cycle, the sPN dissipated nearly 30X more energy than the fPN.

The fact that the fPN undergoes smaller change in volume during inflation is critical for potential applications in space-limited settings (perhaps in procedures such as minimally invasive surgery, or movement through a pipe). The sPN requires outward expansion of its extensible layer to bend, and any restrictions on the space available for its expansion could prevent proper function. The expansion of the extensible layer for the fPN, however, occurs largely within the space between each of its chambers, and thus allows it to move in tighter spaces.

Control of the movement of the actuator is another important parameter characterizing performance. The sPN follows a non-linear relationship between pressure and volume, and is similar to a balloon in that the volume increases but pressure is relatively stable after a threshold pressure(Fig. 2A) ${ }^{[23]}$. This non-linearity of the PV curve would make it difficult or impossible to measure the degree of bending of the SPN by monitoring its pressure. The fPN, however, has a nearly linear PV curve; this quasi-linearity should allow predicting movement by monitoring pressure (Fig. 2B). The fPN therefore has the advantage of providing a complex non-linear output (i.e., a bending motion) with a simple near-linear input (i.e., pressure). 


\section{5 fPNs Fabricated with Different Elastomers}

We measured PV hysteresis curves for a fPN actuator fabricated with a stiffer elastomer (Elastosil M4601, Young's modulus of $7 \mathrm{MPa}$, Shore A hardness 28 ) for the extensible layer. In comparison to Ecoflex 30 (Young's modulus 0.1 MPa, Shore A hardness 00-30), the actuator fabricated with the stiffer elastomer required nearly $8 x$ more pressure, but required $\sim 1.5 x$ less change in volume to bend fully (Fig. 3). The additional volume required by the dPN made of a softer elastomer is a consequence of the outward expansion of its exterior walls. These results suggest that actuators fabricated from softer elastomers exert less force, and bend more slowly for a given rate of inflation than the same actuator made of a stiffer elastomer since additional volume must be transferred to achieve the same degree of bending.

\subsection{Fatigue Testing of fPNs}

An important metric for the performance of elastomeric actuators is the life-span of the device in use; the life-span is limited by fatigue in the material and failure in the adhesive interface ${ }^{[24]}$. We measured the number of full actuation cycles (each cycle consisting of nearly full bending and relaxing) that each type of actuator would withstand before bursting. The sPN (actuated at $0.33 \mathrm{~Hz}$ ) failed, on average, after $\sim 126$ cycles ( $n=11$ sPN with a standard deviation of 274 cycles; Movie S1) and at most after 990 cycles. The large deviation for the failure of the sPN was due primarily to the presence of bubbles (introduced in the elastomer making up the top wall of the extensible layer during fabrication) that weaken the wall, concentrate stress, and lead to the rupture of the extensible layer. Fabricating thicker top walls of the sPN will 

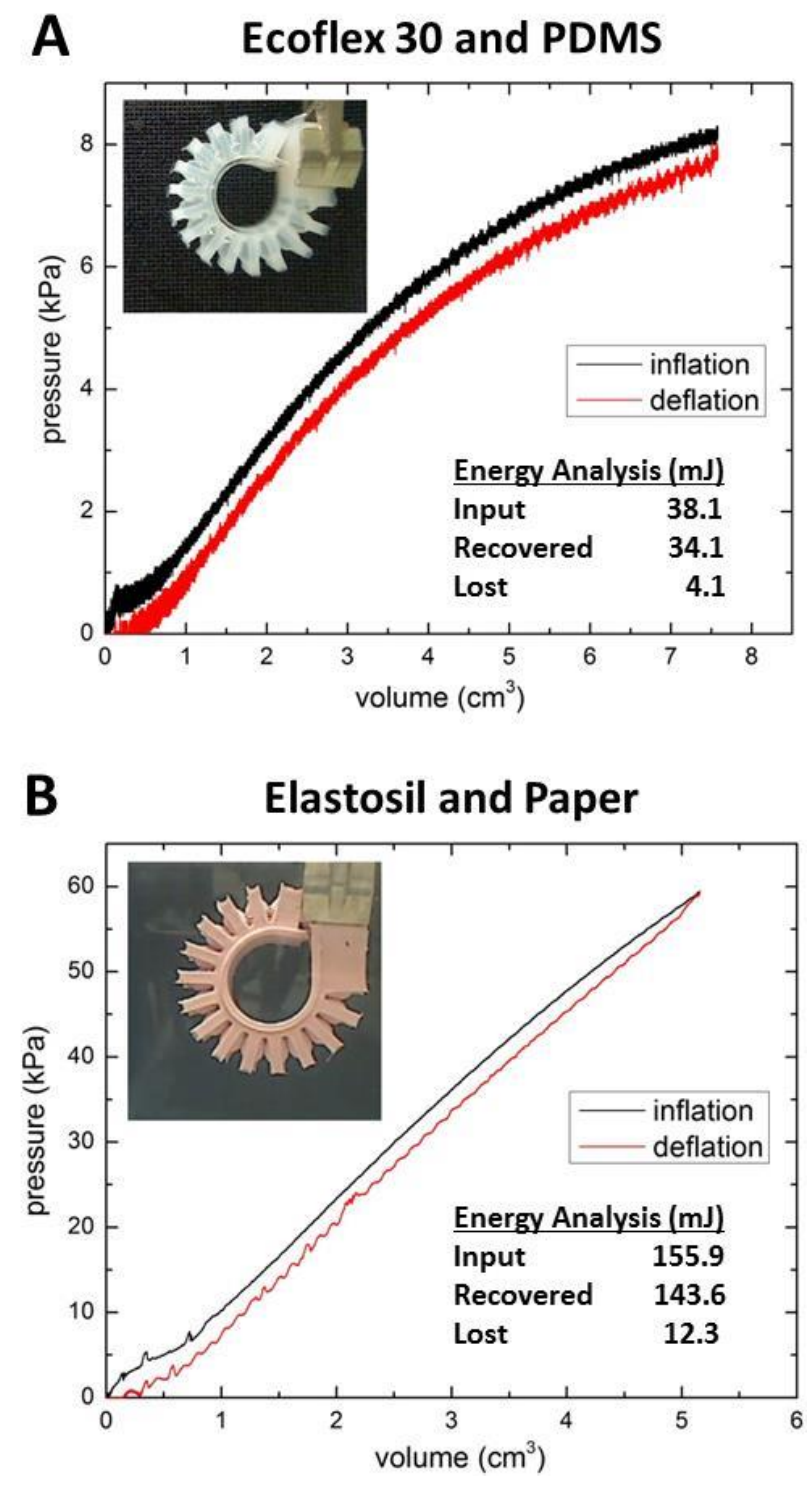

Figure 3. Effect of Elastomer Stiffness. A-B) Pressure-volume hysteresis curves for fPN actuators fabricated with an extensible layer made from Ecoflex 30 and an inextensible layer made from PDMS (A) and an extensible layer made from Elastosil M4601 and inextensible layer made from a composite of Elastosil M4601 and paper fabric (B). 
Figure 4. Fatigue test of fPN actuators. A-C) Pressure-volume hysteresis curves for fPN actuators before and after repeated pneumatic actuation. fPN actuators are made of Elastosil M4601 and paper and are hydraulically actuated to generate the PV curves. 
A fPN actuator after 10k cycles

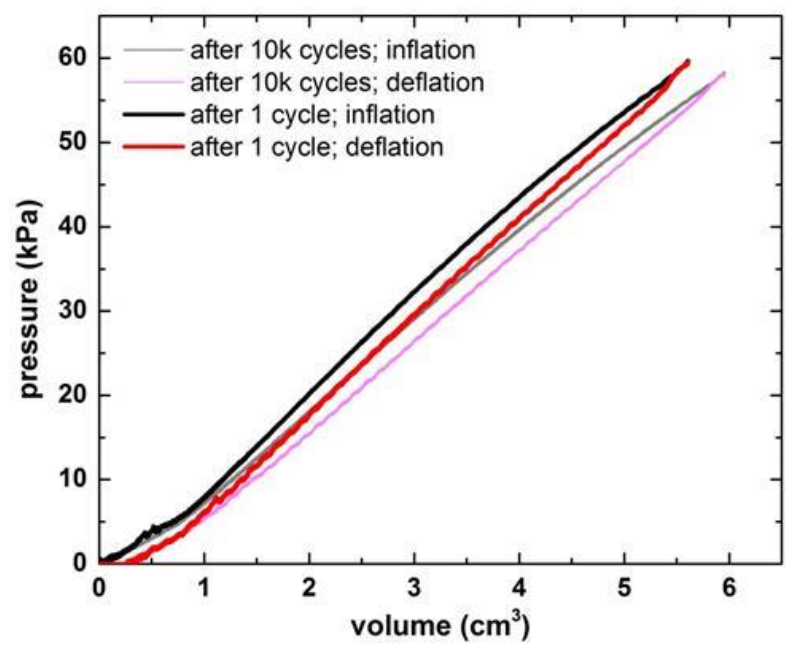

B fPN actuator after 200k cycles

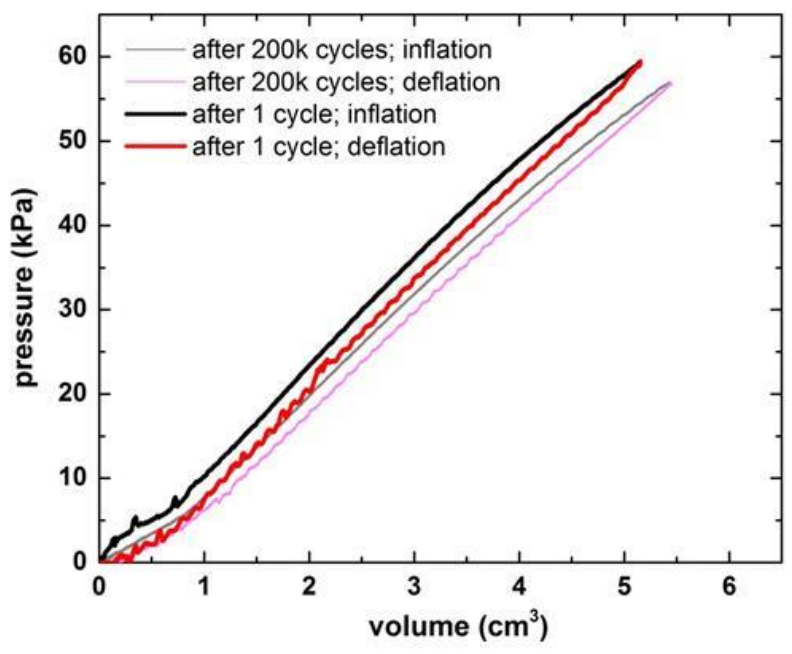

C fPN actuator after $1 \mathrm{M}$ cycles

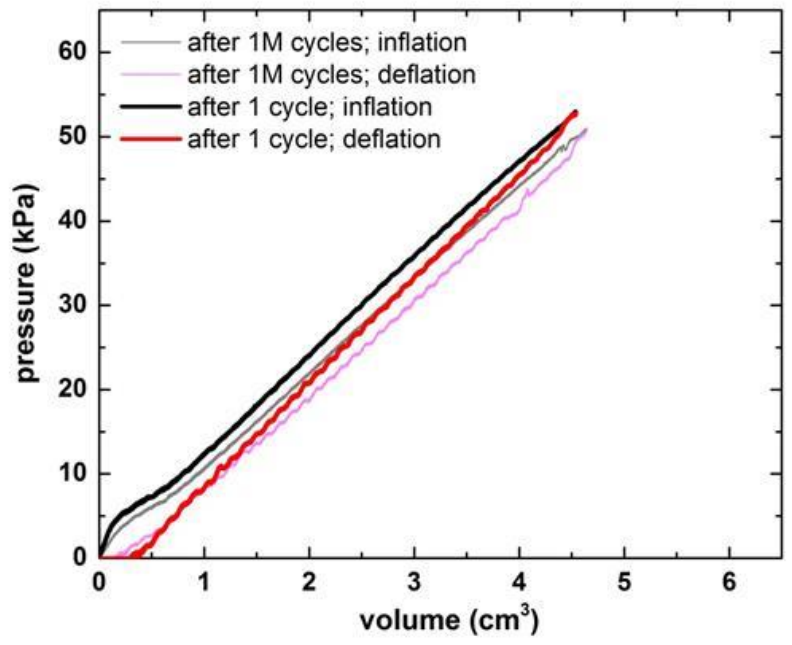


reduce failure due to bubbles, but increase failure by delamination, since higher pressures are required for the actuator to bend fully.

The fPN avoids both modes of failure (interfacial failure and rupture of the extensible layer) by significantly reducing: i) the pressure required for full actuation, ii) the maximum strain in the top wall of the extensible layer required for bending, and iii) bubbles present in the expanding regions of the extensible layer. The fPN thus achieves a life span that is longer than we have been able to measure; the fPN did not fail after cyclic actuation at $2 \mathrm{~Hz}$ for $10^{4}, 2 \times 10^{5}$, and $10^{6}$ cycles of complete actuation.

We assessed fatigue of the material by measuring PV hysteresis curves for three separate fPNs, before and after subjecting them to cyclic testing (Fig. 4). The PV curves do not change substantially over the $10^{6}$ cycles, suggesting that the performance was maintained. The slight decrease in slope, however, suggests that the actuator requires less pressure, but more volume, to bend fully; thus, after many cycles of actuation, the material extends slightly more easily than before the cyclic testing, possibly due to strain of the polymer chains in the elastomer.

\subsection{Analysis of Strain and Force of the SPN and fPN}

To estimate the local strain experienced by both a sPN and fPN, we developed a finiteelement analysis using Abaqus 6.11-Simulia (Dassault Systems). For the FEM, the most critical aspect is properly modeling the non-linear elastomeric behavior of the silicone materials used (Elastosil M4601). We measured stress-strain curves using pieces of cured Elastosil M4601, and 
fit the compression and tensile data to the Yeoh hyperelastic material model in Matlab (Fig. S4). The FEM results show the fPN experiences significantly less ( $2.5 \mathrm{x}$ ) strain (maximum principal or non-deformational) than the SPN for equivalent amplitude of bending (Fig. 2 C-F).

To validate our FEM, we characterized the pressure required to bend a SPN and a FPN with the same number of chambers (15), same height of chambers $(10.5 \mathrm{~mm})$, and same wall thickness $(1 \mathrm{~mm})$ (Fig. S5). We tracked the trajectory of the free end of the actuator using images taken from a camera, and plotted them using a graph that allowed comparison of experimentally observed positions, and positions predicted by the FEM (Fig. S5E, F). The fPN reached its full range of motion ( $360^{\circ}$ bending) at $72 \mathrm{kPa}$ (Fig. S7D); at this pressure, the sPN deflected only $41^{\circ}$ (Fig. S5C).

We also investigated the force these actuators exert for a given pressure to validate the accuracy of the FEM (Fig. S6). Using a transducer (Nano17, 6 axis F/T sensor, ATI industries), we measured the force exerted by the tip of the actuator when the opposite end was fixed in place. As with the actuator trajectories, the forces predicted by the FEM for the SPN and fPN were of good agreement with experimental measurements; this agreement suggests the simulated values of strain are accurate. A fPN applied a force of $\sim 1.4 \mathrm{~N}$ for an actuation pressure of $72 \mathrm{kPa}$ (the pressure that provided full range of bending for the fPN). At the same pressure, a sPN having analogous dimensions applied a force of $\sim 1 \mathrm{~N}$, (i.e., $40 \%$ less than that of the fPN). By increasing the applied pressure (until the actuators rupture), both the SPN and fPN can apply additional force. Changing the stiffness of the elastomer can tune the range of forces 
exerted by both actuators (silicone and polyurethane elastomers with a large range of material properties are commercially available).

\subsection{Rapid Actuation of the SPN and fPN}

To compare how rapidly the sPN and fPN bend, we actuated each with a miniature compressor (BTC-IIS, Parker, Holliss, NH), which delivers compressed air at pressures up to 193 $\mathrm{kPa}$, at a maximum flow rate of $\sim 11 \mathrm{~L} / \mathrm{min}$ (Movie S2 and S3). Using this compressor, on average, the sPN bends fully in 3.3 seconds (standard deviation of $0.54 \mathrm{~s}$ for $\mathrm{N}=7$ actuations) and the fPN bends fully in 130 ms (standard deviation of 8 ms for $\mathrm{N}=7$ actuations).

To determine the maximum frequency the fPN could bend, we used a computer-controlled solenoid valve to pulse pressurized gas for short durations, and then vented the system to equilibrate with atmospheric pressure (Fig. 5A, Movie S4); the head pressure (that is, the pressure set by the regulator) was adjusted to achieve full bending of the fPN. The highest frequency of full bending and relaxing achieved by the fPN, using a $50 \%$ duty cycle (equal durations of pressurizing and venting periods), was $2 \mathrm{~Hz}$ using $76 \mathrm{kPa}$ head pressure (Fig. 5B). Higher frequencies did not allow the actuator to return to its original position. We employed a duty cycle with a shorter pressurization period (50 ms using $448 \mathrm{kPa}$ head pressure) and a longer venting period (200 ms) to achieve an overall higher frequency (4 Hz) of actuation (Fig. 5C, Movie S5).

Figure 5D shows snapshots taken from a movie using a high-speed camera (1000 fps) of the fPN actuator (Movie S6, S7). The fPN showed an interesting and potentially very useful bi-modal 
Figure 5. High-Speed Actuation. A) Images of different positions of the fPN actuator during a full actuation cycle. B) Profile of the internal pressure of the actuator for three frequencies with $50 \%$ duty cycle at pressures that cause full bending of the actuator. C) Profile of the internal pressure for $20 \%$ duty cycle (50 ms pressurization, 200 ms venting) at $413 \mathrm{kPa}$ that yields an actuation frequency of $4 \mathrm{~Hz}$. D) Time-lapse images from high-speed videos of the fPN actuator when actuated with two different pressurization rates. The actuator bends preferentially at its tip for the higher rate and more uniformly for the lower rate. 

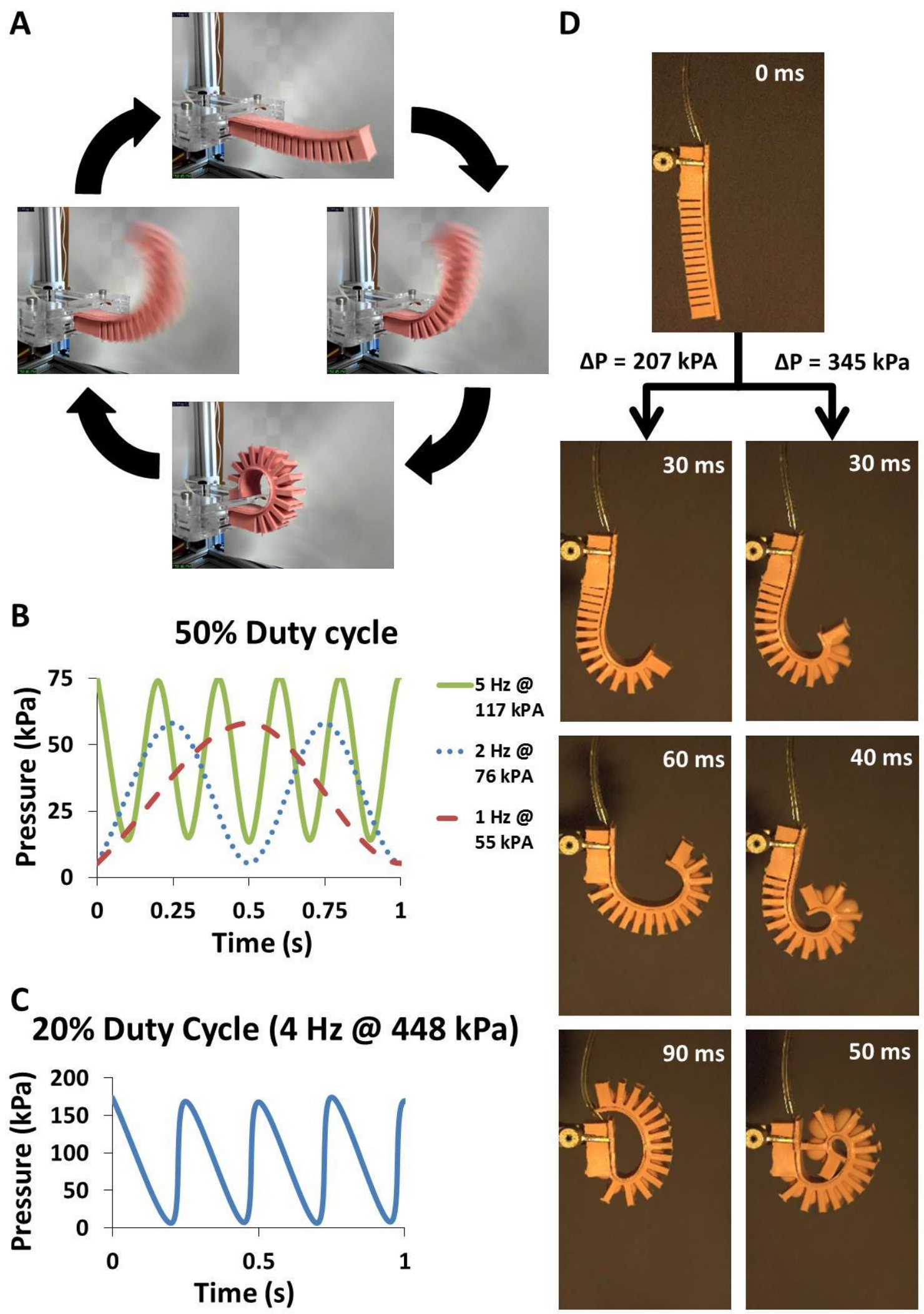
behavior; the change between modes occurs above $\sim 200 \mathrm{kPa}$ head pressure. For slower rates of actuation, the chambers of the fPN inflate relatively evenly, and the actuator bends along a roughly circular trajectory. Above the threshold rate, however, the tip of the actuator bends preferentially, and causes the actuator to curl on itself

\subsection{Pneu-nets Playing a Keyboard}

For maximum utility, soft actuators must have the ability to perform useful tasks rapidly. To provide an example using fPNs, we set four to play an electronic keyboard in a way that mimics (at least distantly) human fingers (Fig. 6A). The actuators require sufficient speed to maintain a desired tempo, and sufficient force $(0.650 \mathrm{~N})$ to depress the keys. The portion of the fPN that contacts the key weighs $\sim 4.5 \mathrm{~g}$ and its gravitational weight can therefore only produce a maximum of $0.045 \mathrm{~N}$ of force. The remaining force $(0.605 \mathrm{~N})$ required to depress a key must be that of the actuator itself (i.e., the internal pressure acting on the actively bending region of the actuator, and the kinetic energy resulting in a transfer of momentum from that bending region of the actuator). We used a solenoid valve to control whether the fPN was pressurized with compressed air (103 $\mathrm{kPa}$ was the maximum pressure of our control system) or vented to the atmosphere; tubing (21 cm long and $3.5 \mathrm{~mm}$ in diameter); a needle (16 gauge) connected the fPN directly to the solenoid valves. The actuator hit the key $100 \mathrm{~ms}$ after opening the valve; to do so, its tip traveled a distance of $\sim 2.6 \mathrm{~cm}$, yielding a momentum of $\sim 1.3 \times 10^{-} \mathrm{N}-\mathrm{s}$ and a force of $\sim 0.005 \mathrm{~N}$. The majority ( $\sim 0.6 \mathrm{~N}$ ) of the force used to depress a key, therefore, results from the pressurization of the fPN. 
Figure 6. Independent Output Control. A) Images from two views of four fPN actuators playing a tune on a digital keyboard. Video is available in the on-line supplemental information. Each fPN was actuated for $75 \mathrm{~ms}$ at $103 \mathrm{kPa}$ (except for repeated notes, which were actuated for 50 ms) and vented for $150 \mathrm{~ms}$. B) Spectrum analysis of the audio file for frequencies between 200 and $500 \mathrm{~Hz}$. 
A

Start Position

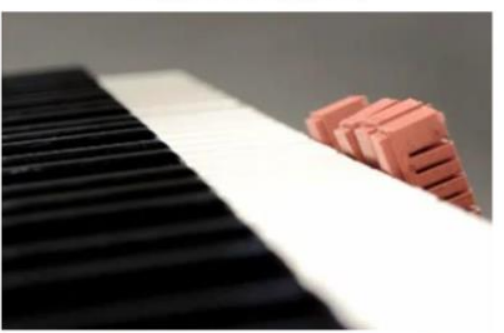

Side

View

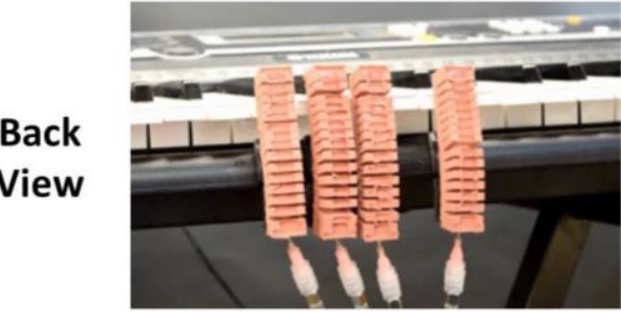

B

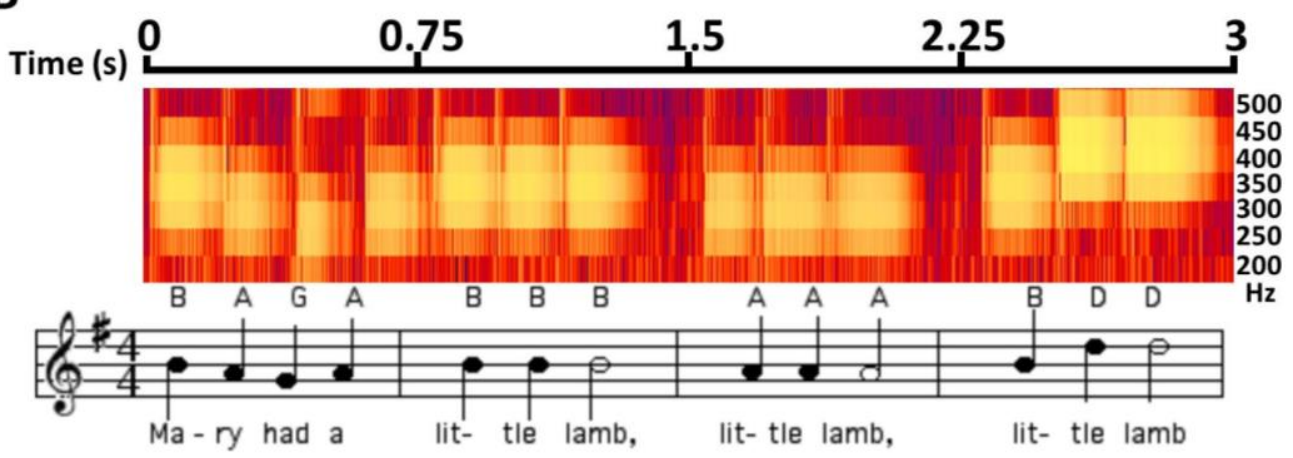


To demonstrate the precision and speed of the fPN actuators, we used four fPNs to play "Mary Had a Little Lamb" on the keyboard (Fig 6B; Movie S8). We fixed these actuators on the side of the keyboard using Velcro, and actuated them with pressurized air using computercontrolled solenoid valves. Playing a note required a period of pressurization of $75 \mathrm{~ms}$ (with a head pressure of $103 \mathrm{kPa}$ ), and a venting period of $150 \mathrm{~ms}$.

Notes played in succession (the same actuator pressurized twice in a row) required a $50 \mathrm{~ms}$ pressurization time, so the actuator would release the key more quickly (alternatively, a longer venting time could have been used, but would have required a slower tempo; Fig. 6A). Figure $6 B$ shows the spectrum analysis of the audio file. The notes played by the fPN match those of the song, and are clearly resolved. This resolution provides one demonstration of the precision of these actuators. The entire two staves play within 6 seconds, and three notes play in as little as 0.4 seconds. To play songs with shorter intervals between notes, we could increase the flow rate of air by using higher pressures (>103 kPa), larger diameter tubing (>3.5 mm), faster mechanisms of venting (e.g., vacuum-driven venting, integrated venting holes directly in the fPNs), and more optimized positioning of the fPNs.

\section{Conclusions}

The performance of a pneu-net can be classified into at least five parameters, i) speed achieved for a given rate of inflation, ii) force exerted for a given pressure, iii) change in volume required for a given degree of bending, iv) number of actuation cycles before failing, and v) correlation between the pressure in the pneu-net and its degree of bending without a load. 
The fPN improves on all these parameters and therefore is better suited for actuating soft robots than the sPN.

Specifically, the improved speed $(25 \mathrm{x})$ and force $(1.4 \mathrm{x})$ of the fPN relative to the SPN will be useful for creating soft robots that move more quickly, and can exert higher forces, than could be achievable with the sPN. Alternatively, one could actuate fPNs with a smaller compressor and obtain performance similar to that generated using a larger compressor and sPNs; the advantage of the system based on the fPN being a reduction in the overall size and power-consumption of the robot (an option particularly useful for untethered applications).

The reduced change in volume ( $8 \times$ smaller than the SPN) minimizes fatiguing of the material, and thus improves the durability of the fPN to such a level that the actuator does not fail within a million cycles of full bending (Fig. 4). This high durability could allow its use in longterm applications, and when high margins of safety are required for high-consequence uses (such as in medical procedures). Furthermore, since the fPN expands into the space between the chambers, the actuator assumes a smaller volume upon inflation, and thus has the potential to enter smaller spaces; this attribute is beneficial for some applications in searchand-rescue and minimally invasive surgery.

In addition to improving the performance (i.e., speed and force) of pneu-nets, we have demonstrated that the rate of actuation (as opposed to the geometry of the actuator) could determine the motion of a pneu-net (Fig. 5D). The bi-modal bending behavior that occurs at high speeds may provide interesting opportunities for control schemes, by allowing at least two different types of motion from a single design, simply by changing the rate of pressurization. 
Further exploration of this rate-dependent behavior may prove fruitful in developing sophisticated soft actuators that can perform tasks that are more useful than playing a keyboard (Fig. 6).

There are two limitations of the specific fPN design described in this work: i) the fPNs bend slightly when gravity acts on the actuator with its bottom layer facing downward, since the chambers connect only at their base, and thus act like a loose hinge (Fig. S5B). Adding material between the chambers or using stiffer materials for the top or bottom layer could prevent this initial bending angle (at the cost of requiring more pressure to actuate the fPN). ii) The chambers of the fPN do not expand uniformly when pressurized above their full bending amplitude due to snap-through instability of the elastomeric material ${ }^{[23]}$. Although this nonlinear behavior may have potential advantages, it also prevents the fPN from exerting forces along its length uniformly. To prevent this behavior, the expansion of the inside walls of the chambers need to be restrained by an inextensible material ${ }^{[3]}$.

\section{Materials and Methods}

Details of fabrication and design for the fPNs are available in the supplementary information. In brief, we used 3D printing to make molds, and soft lithography using those molds to fabricate fPNs. For pneumatic actuation, we pressurized the fPNs with gas supplied from house nitrogen, and vented the chambers on deflation to the atmosphere; computercontrolled solenoid valves regulated actuation of the fPNs. For hydraulic actuation, we used a digital syringe pump that provides a constant flow rate for both inflation and deflation of the 
fPNs. We used both a web-cam and a high-speed camera to observe the movement of the fPNs during actuation.

\section{Acknowledgements}

PV hysteresis curves for comparing the performance of different elastomers and fatigue testing of the actuators and funding for Christoph Keplinger was supported by the Department of Energy (DE-FG02-00ER45852). DARPA (award number W911NF-11-1-0094) supported Robert Shepherd, Sophia Wennstedt, and the development of the actuators and their application to playing the keyboard and rate-dependent bending. We thank the Wyss Institute for Biologically Inspired Engineering for providing support to Bobak Mosadegh and Panagiotis Polygerinos and MRSEC (grant DMR-0820484) for providing funding to Jongmin Shim.

\section{References}

[1] F. Ilievski, A. D. Mazzeo, R. F. Shepherd, X. Chen, G. M. Whitesides, Angew. Chem. Int. Ed. 2011, 50, 1890.

[2] R. V. Martinez, J. L. Branch, C. R. Fish, L. Jin, R. F. Shepherd, R. M. Nunes, Z. Suo, G. M. Whitesides, Adv. Mater. 2013, 25, 205.

[3] R. V. Martinez, C. R. Fish, X. Chen, G. M. Whitesides, Adv. Func. Mat. 2012, 22, 1376.

[4] S. A. Morin, R. F. Shepherd, S. W. Kwok, A. A. Stokes, A. Nemiroski, G. M. Whitesides, Science 2012, 337, 828. 
[5] R. F. Shepherd, F. Ilievski, W. Choi, S. A. Morin, A. A. Stokes, A. D. Mazzeo, X. Chen, M. Wang, G. M. Whitesides, Proc. Natl. Acad. Sci. U.S.A. 2011, 108, 20400.

[6] S. Wakimoto, K. Suzumori, K. Ogura, Adv. Rob. 2011, 25, 1311.

[7] A. A. Stokes, R.F. Shepherd, S.A. Morin, F. Illievski, G. M. Whitesides, Soft Robotics 2013, $1,70$.

[8] L. W. Shi, S. X. Guo, M. X. Li, S. L. Mao, N. Xiao, B. F. Gao, Z. B. Song, K. Asaka, Sensors 2012, 12, 16732.

[9] H. Lee, C. G. Xia, N. X. Fang, Soft Matter 2010, 6, 4342.

[10] M. Otake, Y. Kagami, M. Inaba, H. Inoue, Rob. Auto. Sys. 2002, 40, 185.

[11] E. Brown, N. Rodenberg, J. Amend, A. Mozeika, E. Steltz, M. R. Zakin, H. Lipson, H. M. Jaeger, Proc. Nat. Acad. Sci. U.S.A. 2010, 107, 18809.

[12] R. F. Shepherd, A. A. Stokes, J. Freake, J. Barber, P. W. Snyder, A. D. Mazzeo, L. Cademartiri, S. A. Morin, G. M. Whitesides, Angew. Chem. Int. Ed. 2013, 52, 2892.

[13] C. P. Chou, B. Hannaford, IEEE Trans. Robot. Autom. 1996, 12, 90.

[14] H. T. Lin, G. G. Leisk, B. Trimmer, Bioinspir. Biomim. 2011, 6, 026007.

[15] F. Carpi, S. Bauer, D. De Rossi, Science 2010, 330, 1759.

[16] G. Kofod, W. Wirges, M. Paajanen, S. Bauer, Appl. Phys. Lett. 2007, 90, 081916.

[17] C. Keplinger, M. Kaltenbrunner, N. Arnold, S. Bauer, Proc. Nat. Acad. Sci. U.S.A. 2010, $107,4505$.

[18] M. Shahinpoor, Y. Bar-Cohen, J. O. Simpson, J. Smith, Smart Mat. Struct. 1998, 7, R15.

[19] C. D. Onal, D. Rus, In Proceedings of the 2012 International Conference on Biomedical Robotics and Biomechatronics, 2012. 
[20] E. Palleau, D. Morales, M. D. Dickey, O. D. Velev, Nat. Commun. 2013, 4, 2257.

[21] J. D. W. Madden, N. A. Vandesteeg, P. A. Anquetil, P. G. A. Madden, A. Takshi, R. Z. Pytel, S. R. Lafontaine, P. A. Wieringa, I. W. Hunter, IEEE J. Oceanic Eng. 2004, 29, 706.

[22] N. Cheney, R. MacCurdy, J. Clune, H. Lipson, In Proceedings of the Genetic and Evolutionary Computation Conference, 2013.

[23] T. Li, C. Keplinger, R. Baumgartner, S. Bauer, W. Yang, Z. Suo, J. Mech. Phys. Solids 2013, $61,611$.

[24] T. Zarrin-Ghalami, A. Fatemi, Fatigue Fract. Eng. Mater. Struct. 2013, 36, 270. 